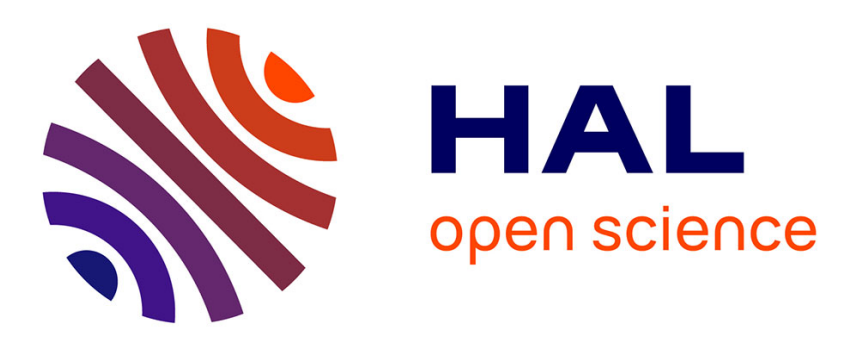

\title{
Modeling the dynamics of interface morphology and crystal phase change in self-catalyzed GaAs nanowires
}

D Wilson, A Sokolovskii, R Lapierre, F Panciera, F Glas, V Dubrovskii

\section{To cite this version:}

D Wilson, A Sokolovskii, R Lapierre, F Panciera, F Glas, et al.. Modeling the dynamics of interface morphology and crystal phase change in self-catalyzed GaAs nanowires. Nanotechnology, 2020, 31 (48), pp.485602. 10.1088/1361-6528/abb106 . hal-03048435

\section{HAL Id: hal-03048435 \\ https://hal.science/hal-03048435}

Submitted on 18 Nov 2021

HAL is a multi-disciplinary open access archive for the deposit and dissemination of scientific research documents, whether they are published or not. The documents may come from teaching and research institutions in France or abroad, or from public or private research centers.
L'archive ouverte pluridisciplinaire HAL, est destinée au dépôt et à la diffusion de documents scientifiques de niveau recherche, publiés ou non, émanant des établissements d'enseignement et de recherche français ou étrangers, des laboratoires publics ou privés. 


\title{
Modeling the dynamics of interface morphology and crystal phase
}

\section{change in self-catalyzed GaAs nanowires}

\author{
D P Wilson ${ }^{1,2}$, A S Sokolovskii ${ }^{2}$, R R LaPierre ${ }^{1,2}$, F Panciera $^{3}$, F Glas $^{3}$, V G Dubrovskii ${ }^{4}$ \\ ${ }^{1}$ Department of Engineering Physics, Centre for Emerging Device Technologies, McMaster \\ University, Hamilton, ON L8S 4L7, Canada \\ ${ }^{2}$ ITMO University, Kronverkskiy pr. 49, 197101 St. Petersburg, Russia \\ ${ }^{3}$ Université Paris-Saclay, CNRS, Centre de Nanosciences et de Nanotechnologies, 91120 \\ Palaiseau, France
}

${ }^{4}$ St. Petersburg State University, Universitetskaya Emb. 13B, 199034, St. Petersburg, Russia

\begin{abstract}
The droplet contact angle and morphology of the growth interface (vertical, tapered or truncated facets) are known to affect the zincblende or wurtzite crystal phase of III-V nanowires grown by the vapor-liquid-solid method. Here, we present a model which describes the dynamics of the morphological evolution in self-catalyzed III-V nanowires in terms of the time-dependent (or length-dependent) contact angle or top nanowire radius under varying material fluxes. The model fits quite well the contact angle dynamics obtained by in situ growth monitoring of self-catalyzed GaAs nanowires in a transmission electron microscope. These results can be used for modeling the interface dynamics and the related crystal phase switching and for obtaining zincblendewurtzite heterostructures in III-V.
\end{abstract}

Keywords: self-catalyzed GaAs nanowires, contact angle, nanowire radius, crystal phase.

\section{Introduction}

Semiconductor nanowires (NWs), particularly III-V NWs, are widely considered as fundamental building blocks for nanoscience and nanotechnology [1-3]. Most III-V NWs are 
synthesized by the vapor-liquid-solid (VLS) method [4] using liquid metal droplets to promote vertical growth. In the case of molecular beam epitaxy (MBE), self-catalyzed VLS growth with gallium droplets [5,6] is commonly employed, which allows one to safely avoid gold contamination, obtain organized arrays of III-V NWs on silicon substrates, modulate at will the NW radius [7-12], and narrow the NW length distributions to sub-Poisson values [13-15]. In situ growth monitoring of the NW growth dynamics inside a transmission electron microscope (TEM) [16-19] plays an important role in understanding the VLS process and controlling the crystal phase of III-V NWs, which can be either cubic zincblende (ZB) or hexagonal wurtzite (WZ) depending on the growth conditions [20].

Recent in situ TEM data on MBE growth of self-catalyzed GaAs NWs [19] clearly reveal the droplet contact angle as the main parameter governing the crystal phase selection. It has been demonstrated that the ZB phase forms at small $\left(<100^{\circ}\right)$ and large $\left(>125^{\circ}\right)$ contact angles, whereas pure WZ phase is observed for intermediate contact angles. WZ NWs are restricted by vertical sidewalls, whereas ZB NWs taper at small contact angles $\left(<100^{\circ}\right)$ or develop a truncated edge at their top at large contact angles $\left(>125^{\circ}\right)$. Above a certain maximum contact angle, which was estimated at around $130^{\circ}$ in Ref. [11], ZB NWs start to develop inverse tapering. These trends have been explained by a stationary model based entirely on the surface energetics $[19,21]$, and related to the bi-stability of the contact angle under stationary conditions [11]. The droplet contact angle can be finely tuned by changing the group III and V fluxes [11,17,19], which gives a simple tool for crystal phase switching and obtaining ZB-WZ heterostructures in GaAs NWs. However, a more detailed theoretical description of the interface dynamics under varying material fluxes is missing. Consequently, here we present a model which describes the time-dependent contact angle for vertical NWs, or the top NW radius (at smaller or larger contact angles) for tapered or inversetapered NWs. The model fits quite well the data on the evolution of the contact angle in selfcatalyzed GaAs NWs [19] and should be useful for the fine tuning of the NW interface morphology and the related crystal phase in a wide range of VLS III-V NWs. 


\section{Model}

In self-catalyzed growth of III-V NWs, the number of group III atoms in the droplet $N_{3}$ is given by the ratio of its volume (with neglect of group $\mathrm{V}$ atoms due to their extremely low concentration) over the elementary volume per atom in liquid $\Omega_{L}$

$N_{3}=\frac{\pi R^{3} f(\beta)}{3 \Omega_{L}}$

Here, $R$ is the top radius of the cylindrical NW, which equals the radius of the droplet base, and

$f(\beta)=\frac{(1-\cos \beta)(2+\cos \beta)}{(1+\cos \beta) \sin \beta}$

is the geometrical function relating the volume of spherical cap to the cube of its base through the droplet contact angle $\beta$, with $d f / d \beta=3 /(1+\cos \beta)^{2}$.

According to Ref. [22], the change in $N_{3}$ is described by the material transport equation

$\frac{d N_{3}}{d t}=\frac{\pi R^{2}}{\Omega_{S}}\left[\frac{2 \lambda_{3} v_{3} \sin \alpha_{3}}{\pi R} \frac{\left(1-\theta_{l f}\right)\left[\cosh \left(L / \lambda_{3}\right)-1\right]+\theta_{s f}-\theta_{l f}}{\sinh \left(L / \lambda_{3}\right)}-\left(\chi_{5} v_{5}-\chi_{3} v_{3}\right)\right]$

Here, $\Omega_{S}$ is the elementary volume per III-V pair in solid, $\theta_{s f}=n_{s} / n_{f}$ and $\theta_{l f}=n_{l} / n_{f}$ are the ratios of the group III adatom concentration $(n)$ on the substrate surface (s) to the side facets of the NW (f), and at the liquid-solid interface (1) to the side facets, respectively. They can be related to the corresponding chemical potential differences as discussed in Refs. [23] and [24]. $L$ denotes the NW length, and $\lambda_{3}$ is the collection length of group III adatoms on the NW side facets. The term proportional to $\left(1-\theta_{l f}\right)$ in the $1 / R$ diffusion flux in Eq. (3) stands for group III atoms collected by the NW sidewalls. The term proportional to $\theta_{s f}-\theta_{l f}$ describes the diffusion flux from the substrate surface. The diffusion flux is positive when $\theta_{l f}<1$ and $\theta_{s f}>\theta_{l f}$, meaning that more group III adatoms enter the droplet from the NW sidewalls than escape from the droplet. Negative diffusion fluxes correspond to reverse diffusion of group III atoms from the droplet onto the NW sidewalls [23]. Very importantly, the last bracket term in Eq. (3) is proportional to the group V to III influx imbalance, $\chi_{5} v_{5}-\chi_{3} v_{3}$, with $v_{k}$ for $k=3,5$ as the atomic fluxes of group III and $\mathrm{V}$ elements onto the surface (measured in $\mathrm{nm} / \mathrm{s}$ ). The droplet volume will self-equilibrate to a certain 
stationary value when $\chi_{5} v_{5}>\chi_{3} v_{3}[8,9,12]$, while in the opposite case it will only grow [7,11]. The geometrical functions $\chi_{k}\left(\alpha_{k}, \beta\right)$ in directional MBE growth depend on the beam angles $\alpha_{k}$ of group III and V species with respect to the substrate normal and the contact angle $\beta$ according to Ref. [25].

In many cases [8,26,27], the axial growth rate of self-catalyzed III-V NWs is proportional to the incoming flux of group $\mathrm{V}$ atoms

$\frac{d L}{d t}=\chi_{5}\left(\alpha_{5}, \beta\right) v_{5}$

To model experimental data, we assume that $L / \lambda_{3} \gg 1$, in which case Eq. (3) contains only the diffusion flux of group III adatoms from the NW sidewalls. Expressing $d N_{3} / d t$ through $d R / d t$ and $d \beta / d t$ from Eqs. (1) and (2) and eliminating time by combining Eqs. (3) and (4), after some simple manipulations we obtain the dynamics of the NW top radius and contact angle versus the NW length $L$ in the form

$f(\beta) \frac{d R}{d L}+\frac{R}{(1+\cos \beta)^{2}} \frac{d \beta}{d L}=a+\frac{\rho}{R}$.

Here, the coefficients are given by

$a=\frac{\Omega_{L}}{\Omega_{S}}\left(\frac{\chi_{3} v_{3}}{\chi_{5} v_{5}}-1\right), \rho=\frac{\Omega_{L}}{\Omega_{S}} \frac{2 \lambda_{3} \sin \alpha_{3}\left(v_{3}-v_{l}\right)}{\pi \chi_{5} v_{5}}$.

Clearly, the coefficient $a$ describes the contribution from the direct impingement of group III atoms minus their sink due to NW growth, while $\rho$ gives the diffusion flux of group III adatoms. $v_{l}$ is the reverse diffusion flux from the droplet onto the NW sidewalls such that $v_{l} / v_{3}=n_{l} / n_{f}$. The total diffusion flux is positive when $v_{3}>v_{l}$ and negative otherwise. In our MBE system (in situ TEM), Ga flux is perpendicular to the NW axis and As flux parallel to it, therefore

$\alpha_{3}=\pi / 2, \chi_{3}=\frac{2 \beta-\sin (2 \beta)}{2 \sin ^{2} \beta}, \chi_{5}=\frac{1}{\sin ^{2} \beta}$.

According to Eq. (5), the volume of the catalyst droplet may change by changing either the NW top radius $R$ or the droplet contact angle $\beta$. The recent data on the morphology of selfcatalyzed GaAs NWs $[11,19,21]$ can be summarized as follows. Under the stationary conditions corresponding to a fixed V/III ratio, GaAs NWs show two stable contact angles, $\beta_{\min }$ around 90- 
$100^{\circ}$ and $\beta_{\max }$ around $130-140^{\circ}$, corresponding to shrinking or extending their top radius $R$ in the ZB phase. Below $\beta_{\text {min }}$, the NW is ZB and has narrowing (n) facets of angle $\varphi_{n}$ while above $\beta_{\max }$ the NW is ZB and has widening (w) facets of angle $\varphi_{w}$ (case 1). Between these angles the NW has vertical sidewalls (case 2) and is mainly WZ except above a critical contact angle $\beta_{\text {crit }}$ around $125^{\circ}[19]$. These contact angles are semi-stable, meaning that if the change in flux is not "too fast" the contact angle may remain constant at either of these angles (case 3). We define as "too fast" a flux change which produces a change in radius $d R / d L>\left|\tan \varphi_{k}\right|$. These cases are described by Case 1:

$R=R_{0}-\tan \varphi_{k}\left(L-L_{0}\right)$

$\frac{d \beta}{d L}=\frac{(1+\cos \beta)^{2}}{R}\left[\left(a+\frac{\rho}{R}\right)+f(\beta) \tan \varphi_{k}\right]$

with $L_{0}$ and $R_{0}$ the initial length and radius of the widening or narrowing NW segment.

Case 2:

$\frac{d \beta}{d L}=\left(a+\frac{\rho}{R}\right) \frac{(1-\cos \beta)^{2}}{R_{0}}$

at a constant radius $(d R / d L=0)$, which is the limiting case of Eq. (9) for vertical NW sidewalls $\left(\varphi_{k}=0\right)$.

Case 3:

$\frac{d R}{d L}=\frac{(a+\rho / R)}{f(\beta)}$

at a constant $\beta=\beta_{\min }$ or $\beta=\beta_{\max }$, as in Refs. [7,8].

IF we ignore the $\beta$ dependence in the geometrical coefficients $\chi_{k}$, we obtain by integration Case 1:

$f(\beta)=\frac{1}{(1-c x)^{3}}\left\{f\left(\beta_{0}\right)-\frac{a}{c R_{0}}\left[(1-c x)^{3}-1\right]-\frac{3 \rho}{2 c R_{0}^{2}}\left[(1-c x)^{2}-1\right]\right\}$.

Case 2:

$f(\beta)=f\left(\beta_{0}\right)+3\left(\frac{a}{R_{0}}+\frac{\rho}{R_{0}^{2}}\right) x$, 
where for brevity we have let $x=L-L_{0}$ and $c=\tan \varphi_{k} / R_{0}$. Integrating Eq. (11), in Case 3 we find:

$x=\frac{f(\beta)}{a}\left[R-R_{0}-\frac{\rho}{a} \ln \left(\frac{R+\rho / a}{R_{0}+\rho / a}\right)\right]$,

which agrees with the result of Refs. [7,8].

\section{Results and discussion}

The data for the contact angle and the NW radius dynamics under varying group III and V fluxes, obtained by in situ monitoring of MBE growth of self-catalyzed GaAs NWs grown inside the TEM NanoMax at $420{ }^{\circ} \mathrm{C}$ [19], are shown in Figure 1. To fit them, we solve all equations numerically using Python 3.7. The solutions to the ordinary differential equations (Eqs. (8) to (10)) are found using a Runge-Kutta method [28], whereas the approximate forms of the equations (Eqs. (12) to (14)) are solved by finding the zeros of the equation using the secant method [29]. At the end of each solver step, the conditions for the case being solved are checked. If the solution no longer meets the criteria, the solver is switched to the appropriate case and uses the previous solution as the new starting conditions. The best fit is found for the parameters $\lambda_{3}, v_{5}, v_{l}$ and $\beta_{\max }$ by minimizing the sum of squared residuals. We use a global differential evolution solver to narrow down the parameter space [30] and then refine the solution locally with a Nelder-Mead minimization algorithm [29].

The data set of Ref. [19] results from several changes to the Ga and As fluxes. To set our numerical results in the context of the experiment we use the values of the Ga flux, $v_{3}$, stated in Ref. [19] $(0.15 \mathrm{~nm} / \mathrm{s}$ or $0 \mathrm{~nm} / \mathrm{s})$. The effective As flux, $v_{5}$, can differ from the direct flux due to reflections within the MBE [26] so these values are solved for rather than taken from Ref. [19]. However, we assume that sections with the same direct flux ratio in the experiment should produce the same values of $v_{5}$ and $v_{l}$ and constrain our fitting algorithm to ensure this is the case. Changes in flux are assumed to be instantaneous. The best fits shown in Figure 1 are obtained with the 
parameters summarized in Table 1 for each NW section corresponding to different As and Ga fluxes.
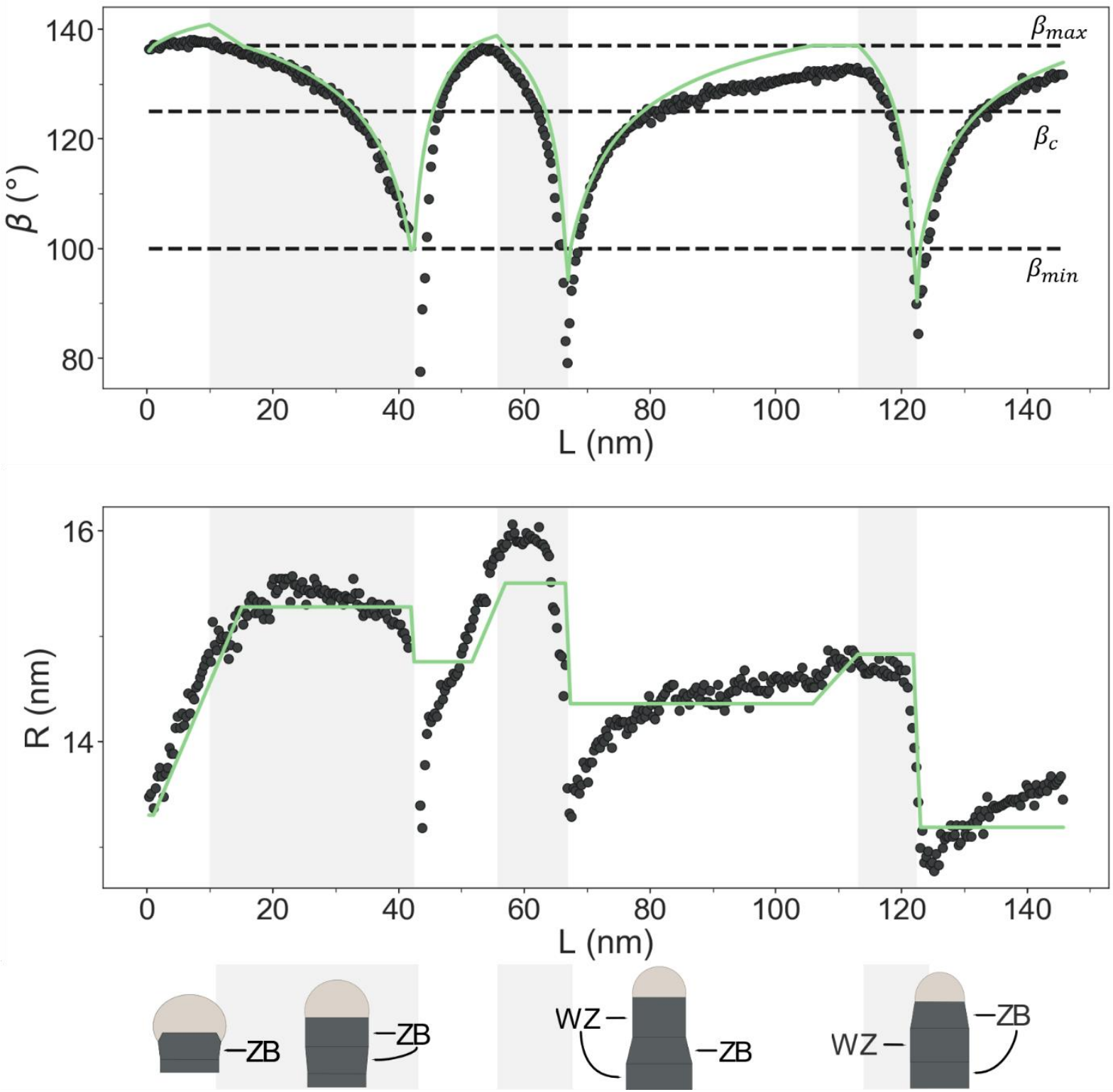

Figure 1. Experimental data (symbols) and best fit (lines) of the contact angle (upper panel) and radius (lower panel) dynamics in a single self-catalyzed GaAs NW. The fit is obtained using the parameters given in Table 1 to find the solution. The alternating blocks of white and shaded background indicate changes in the V/III ratio as shown in Table 1 . The values of $\beta_{\max }$ and $\beta_{\min }$ are $137^{\circ}$ and $100^{\circ}$, respectively, as indicated by dashed lines. The values of $\varphi_{w}$ and $\varphi_{n}$ are $-8^{\circ}$ and $55^{\circ}$, respectively, as measured from the images of Ref. [19]. The diagrams in the bottom panel show the NW shape and crystal phase. Transitions from the $\mathrm{WZ}$ to the $\mathrm{ZB}$ phase occur when the contact angle becomes larger than $\beta_{c}=125^{\circ}$, with vertical NW sidewalls and truncated top above this value, or smaller than $\beta_{\min }=100^{\circ}$, with tapered NW morphology and flat top below this value [19]. 
Table 1 Values of the parameters used to produce the fits to the data

\begin{tabular}{|c|c|c|c|c|}
\hline & Inputs & \multicolumn{3}{|c|}{ Parameters } \\
\hline Segment & $v_{3}(\mathrm{~nm} / \mathrm{s})$ & $\lambda_{3}(\mathrm{~nm})$ & $v_{5}(\mathrm{~nm} / \mathrm{s})$ & $v_{l}(\mathrm{~nm} / \mathrm{s})$ \\
\hline $\mathrm{A}, \mathrm{C}$ & 0.15 & \multirow{3}{*}{461} & 0.1000 & 0.06167 \\
\hline $\mathrm{B}$ & 0.15 & & 0.2318 & 0.2183 \\
\hline $\mathrm{nnnn}, \mathrm{N}$ & 0 & 0.8989 & 0.7847 \\
\hline $\mathrm{nnnny}$ & & & 0.4736 & 0.02472 \\
\hline
\end{tabular}
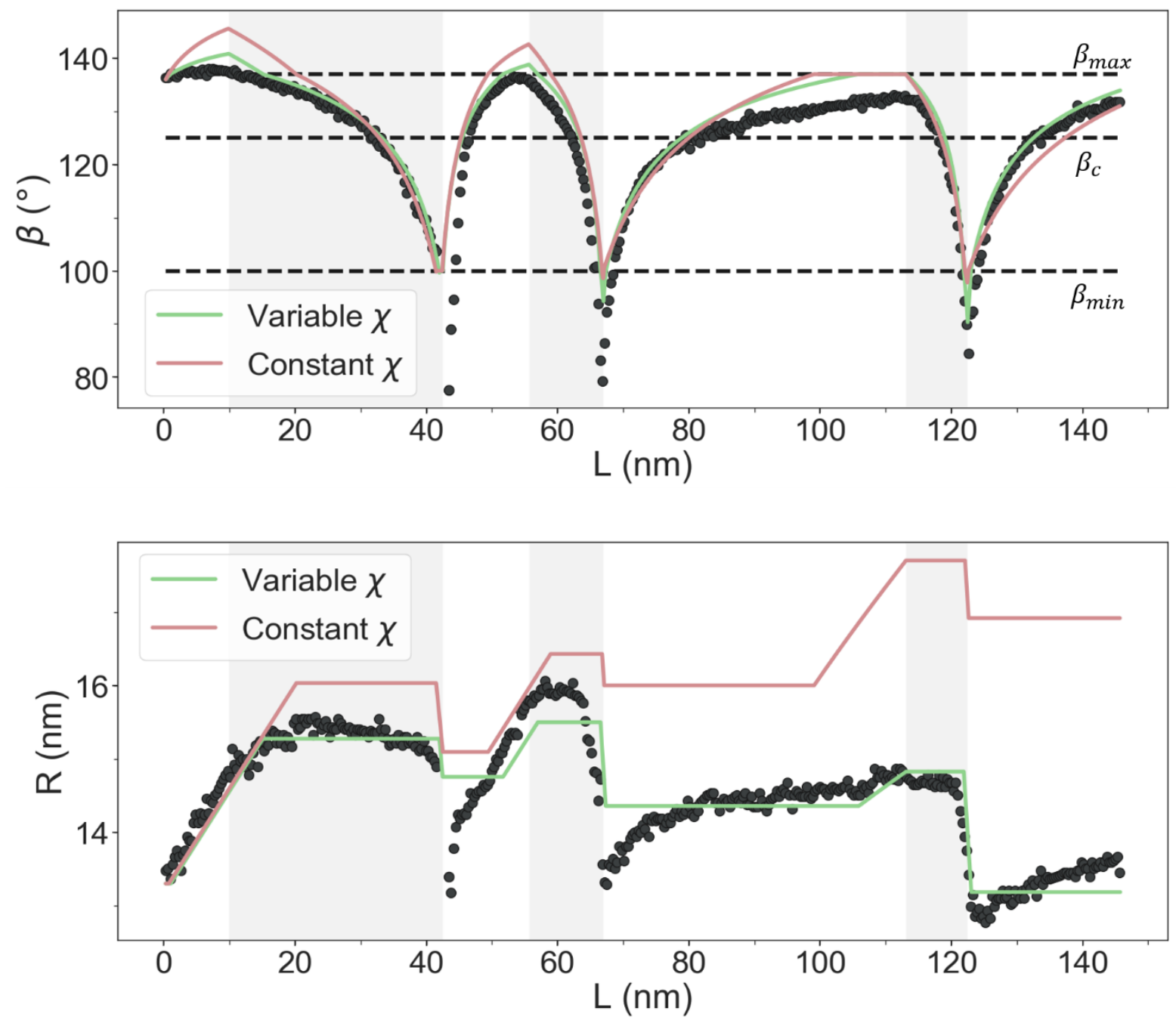

Figure 2. The solutions from solving the equations with $\chi$ dependent on $\beta$ as compared to using constant $\chi$ values. The solution shown for variable $\chi$ is the same as that in Fig. 1. Both solutions use the parameters given in Table 3. The constant value of $\chi_{3}$ and $\chi_{5}$ were set to 0.808 and 1.132 , respectively, corresponding to $\beta=110^{\circ}$. We choose $110^{\circ}$ because it falls in the middle of the range of contact angles present in this study. While the plots are similar for $\beta$, small differences compound as $L$ increases which results in the plots of $R$ diverging from one another. 
Figure 2 shows a comparison of the solutions to Eqs. (8) to (11) with the approximations at constant $\chi_{3}$ and $\chi_{5}$ values given by Eqs. (12) and (13) for the same parameters given in Table 1. We can see that the fits obtained for the contact angle dynamics are only slightly worse than those obtained from the general equations. However, the constant $\chi$ model cannot reproduce the dynamics of NW radius. Figure 3 shows the inverted $v_{3} / v_{5}$ and $\left(v_{3}-v_{l}\right) / v_{5}$ ratios corresponding to the fitting parameters given in Table 1 versus the V/III flux ratios as presented in Ref. [19]. The inverted $v_{3} / v_{5}$ ratios obtained from the fits are not identical to the V/III ratios of Ref. [19] but remain in the range on the order of unity. For NW segments B, D and F corresponding to the droplet shrinkage, the values $v_{3}-v_{l}$ are negative (even if $v_{3}$ is non-zero as in segment $\mathrm{B}$ ) and so are the $\left(v_{3}-v_{l}\right) / v_{5}$ ratios.

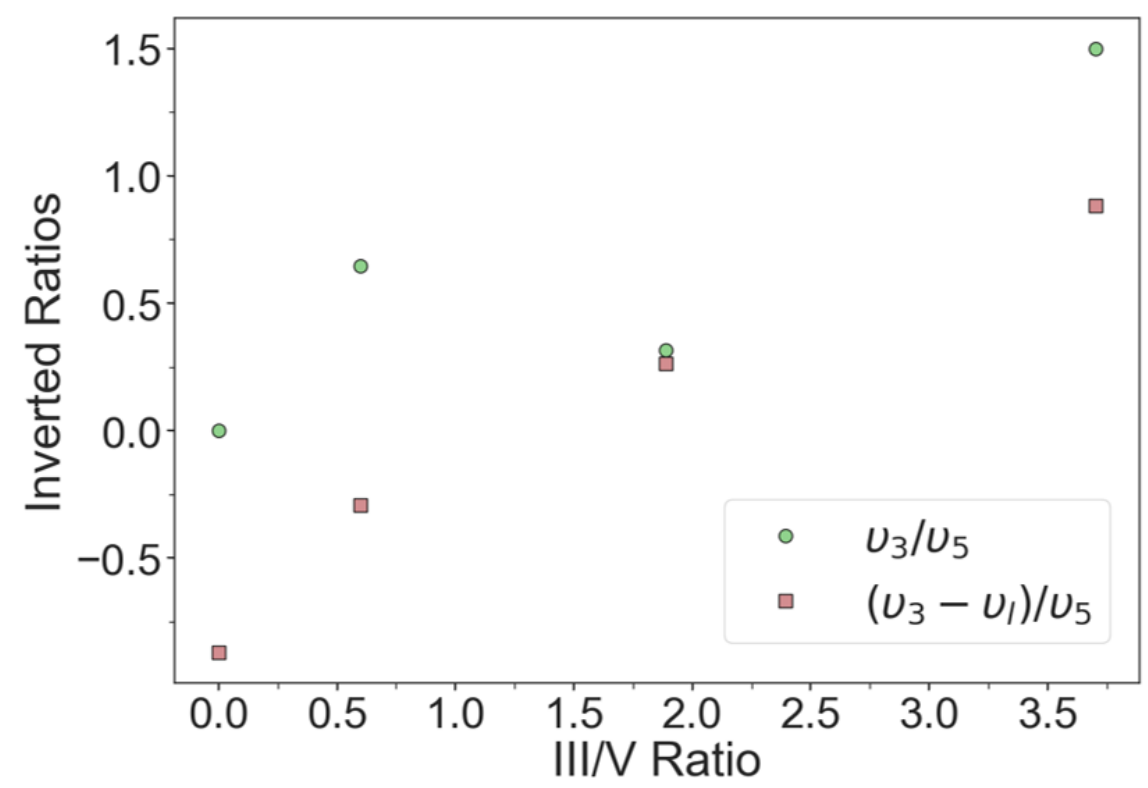

Figure 3. The relationship between the inverted V/III ratios numerically determined for the solved parameters and the V/III flux ratios as presented in Ref. [19]. Both the V/III ratios and the V/III ratios with reverse diffusion are shown.

Let us now analyze these results and the parameter values used to obtain best fits to the data. The taper angles for widening and narrowing side facets were obtained from the TEM images of Ref. [19]. The contact angle changes under varying material fluxes are well-fitted with a fixed collection length of gallium adatoms of $461 \mathrm{~nm}$ regardless of the V/III flux ratio. This relatively 
small value (compared, for example, to $750 \mathrm{~nm}$ in Ref. [8]) should be due to the low growth temperature of $420^{\circ} \mathrm{C}$, because the surface diffusion is thermally activated [3]. At a fixed $\lambda_{3}$, the main parameter influencing the fits is the reverse diffusion flux of $\mathrm{Ga}$ from the droplet onto the NW sidewalls, $v_{l}$. It is well known that the ratio $v_{l} / v_{3}=n_{l} / n_{f}$, which directs the Ga diffusion flux to the droplet at $v_{l} / v_{3}<1$ or from the droplet at $v_{l} / v_{3}>1$, changes very substantially when altering the V/III flux ratio [24]. Here, we find that $v_{l} / v_{3}$ is noticeably lower than unity (on the order of 0.2-0.4) in segments A, C, E and G corresponding to the droplet inflation steps and ultimately to large droplets with the contact angles within the range of $125-140^{\circ}$ and pure $\mathrm{ZB}$ phase of GaAs NWs. This yields high Ga diffusion fluxes from the NW sidewalls to the droplet which are several times larger than the direct vapor flux of Ga. Conversely, in segments B, D and F, the Ga diffusion flux is directed from the droplet down to the substrate. This reverse diffusion is the main mechanism of the droplet shrinkage to a contact angle of $100^{\circ}$ or even less, and the crystal phase of GaAs NWs becomes ZB again. As a general conclusion, the possibility to rapidly redirect the diffusion flux of Ga to or from the droplet by changing the V/III flux ratio allows for a more rapid change of the droplet contact angle which determines the crystal phase and consequently thinner ZB/WZ heterostructures.

We note that the changes of the contact angle shown in Figure 1 are highly non-linear, particularly for small $\beta$ below $\beta_{\text {min }}$. The amplitude of the radius oscillations obtained from in situ data (symbols in Figures 1 and 2$)$ is very small $(<3 \mathrm{~nm})$. However, the variations of the measured radius are non-linear, while theoretical curves are linear due to this very small amplitude of oscillations. We found it impossible to exactly fit in situ data for the contact angle and the NW radius dynamics simultaneously using the current model. Figures 1 and 2 show that, when we solve for the best fit of the contact angle and radius simultaneously, there is a discrepancy in the measured and modelled radius changes. This may be partly due to the radial growth, which is not included in the model but affects the radius of WZ NW sections during the increase of the contact angle. We use cylindrical NW geometry and spherical cap droplet geometry, while in the real NWs 
the liquid droplet is constrained to rest on top of a hexagonal shape. Finally, the small and large contact angles were estimated in Refs. $[11,19,21]$ from surface energy considerations and under stationary material fluxes, while the data of Ref. [19] were obtained with abrupt changes of the fluxes. Despite these complicated factors, the current model fits very accurately the contact angle crossing the two critical angles $\left(\beta_{\min }=100^{\circ}\right.$ and $\left.\beta_{c}=125^{\circ}\right)$ for the crystal phase changes.

In conclusion, our model describes fast changes of the droplet contact angle under variable fluxes of group III and V elements. We considered self-catalyzed VLS growth of III-V NWs by MBE. However, generalization of this approach to Au-catalyzed VLS growth of III-V NWs requires only a slight modification. The crystal phase change is determined by the dynamics of the droplet contact angle. In the case of GaAs NWs, there is a wide range of the contact angles in which the NWs grow in the WZ phase with vertical sidewalls. As discussed in Ref. [19], the best point for sharp ZB-WZ phase change is around the critical contact angle of $125^{\circ}$, because it occurs without changing the NW radius and does not require too rapid droplet shrinkage under excessive V/III flux ratio. The situation may be different for GaP NWs, which seem to maintain a stable contact angle around $123^{\circ}$ in the ZB phase according to Refs. [12] and [21]. In this case, a ZB-WZ phase transition may require tapering. It will be interesting to consider in more detail the droplet dynamics in doped GaAs and other III-V NWs where the introduction of doping impurities may change the surface energetics and lead to the formation of regular twinning superlattices [31]. We plan to study the interface dynamics of other III-V NWs using a similar approach. We will also refine the model to include the radial growth on the NW sidewalls, which occurs after the crystal phase is decided. Overall, our approach should be applicable to a wide range of III-V materials and epitaxy techniques, and further used for developing the growth protocols to form ZB-WZ NW heterostructures in a controllable manner.

\section{Acknowledgements}

ASS and VGD thank the Russian Foundation for Basic Research for the financial support under grants 18-02-40006, 19-52-53031, 20-52-16301, and 20-02-00351. DPW and RRL thank 
the Natural Sciences and Engineering Research Council of Canada under Grant RGPIN-201804015 and NSERC CREATE TOP-SET under Grant 497981-2017. FG and VGD acknowledge the support of the International Research Project "Physics of nanostructures and innovative devices based on compound semiconductors" (PHYNICS) established between Centre National de la Recherche Scientifique (CNRS) and the Russian Foundation for Basic Research. We acknowledge the ANR (French National Research Agency) for funding the NanoMAX ETEM through the TEMPOS grant, project number 10-EQPX-0050.

\section{References}

[1] Yang P, Yan R and Fardy M 2010 Semiconductor nanowires: what's next? Nano Lett. 101529 1536

[2] Zhang A, Zheng G and Lieber C M 2016 Nanowires: building blocks for nanoscience and nanotechnology (Berlin: Springer)

[3] Dubrovskii V G 2015 Theory of VLS growth of compound semiconductors Semiconductors and Semimetals ed A Fontcuberta i Morral et al vol 93 (New York: Academic) pp 1-78

[4] Wagner R S and Ellis W C 1964 Vapor-liquid-solid mechanism of single crystal growth Appl. Phys. Lett. 489

[5] Colombo C, Spirkoska D, Frimmer M, Abstreiter G and Fontcuberta i Morral A 2008 Gaassisted catalyst-free growth mechanism of GaAs nanowires by molecular beam epitaxy Phys. Rev. B 77155326

[6] Jabeen F, Grillo V, Rubini S and Martelli F 2008 Self-catalyzed growth of GaAs nanowires on cleaved Si by molecular beam epitaxy Nanotechnology 19275711

[7] Priante G, Ambrosini S, Dubrovskii V G, Franciosi A and Rubini S 2013 Stopping and resuming at will the growth of GaAs nanowires Cryst. Growth Des. 13 3976-3984

[8] Dubrovskii V G, Xu T, Díaz Álvarez A, Plissard S, Caroff P, Glas F and Grandidier B 2015 Self-equilibration of the diameter of Ga-catalyzed GaAs nanowires Nano Lett. 15 5580-5584 
[9] Tersoff J 2015 Stable self-catalyzed growth of III-V nanowires Nano Lett. 15 6609-6613

[10] Matteini F, Dubrovskii V G, Rüffer D, Tütüncüoğlu G, Fontana Y and Fontcuberta i Morral A 2015 Tailoring the diameter and density of self-catalyzed GaAs nanowires on silicon Nanotechnology 26105603

[11] Kim W, Dubrovskii V G, Vukajlovic-Plestina J, Tütüncüoglu G, Francaviglia L, Güniat L, Potts H, Friedl M, Leran J B and Fontcuberta i Morral A 2018 Bi-stability of contact angle and its role in achieving quantum-thin self-assisted GaAs nanowires Nano Lett. 18 49-57

[12] Leshchenko E D, Kuyanov P, LaPierre R R and Dubrovskii V G 2018 Tuning the morphology of self-assisted GaP nanowires Nanotechnology 29225603

[13] Glas F and Dubrovskii V G 2017 Self-narrowing of size distributions of nanostructures by nucleation antibunching Phys. Rev. Materials 1036003

[14] Koivusalo E S, Hakkarainen T V, Guina M and Dubrovskii V G 2017 Sub-Poissonian narrowing of length distributions realized in Ga-catalyzed GaAs nanowires Nano Lett. 17 53505356

[15] Tauchnitz T, Berdnikov Y, Dubrovskii V G, Schneider H, Helm M and Dimakis E 2018 A simple route to synchronized nucleation of self-catalyzed GaAs nanowires on silicon for subPoissonian length distributions Nanotechnology 29504004

[16] Wen C-Y, Tersoff J, Hillerich K, Reuter M C, Park J H, Kodambaka S, Stach E A and Ross F M 2011 Periodically changing morphology of the growth interface in $\mathrm{Si}, \mathrm{Ge}$, and GaP nanowires Phys. Rev. Lett. 107025503

[17] Jacobsson D, Panciera F, Tersoff J, Reuter M C, Lehmann S, Hofmann S, Dick K A and Ross F M 2016 Interface dynamics and crystal phase switching in GaAs nanowires Nature $\mathbf{5 3 1} 317$

[18] Harmand J C, Patriarche G, Glas F, Panciera F, Florea I, Maurice J L, Travers L and Ollivier Y 2018 Atomic step flow on a nanofacet Phys. Rev. Lett. 121166101

[19] Panciera F, Baraissov Z, Patriarche G, Dubrovskii V G, Glas F, Travers L, Mirsaidov U and Harmand J C 2020 Phase selection in self-catalyzed GaAs nanowires. Nano Lett. 20 1669-1675 
[20] Glas F, Harmand J C and Patriarche G 2007 Why does wurtzite form in nanowires of III-V zinc-blende semiconductors? Phys. Rev. Lett. 99146101

[21] Dubrovskii V G, Sibirev N V, Halder N N and Ritter D 2019 Classification of the morphologies and related crystal phases of III-V nanowires based on the surface energy analysis J. Phys. Chem. C 123, 18693-18701

[22] Dubrovskii V G 2019 Stabilization of the morphology and crystal phase in ensembles of selfcatalyzed GaAs nanowires Phys. Stat. Sol. RRL 131900301

[23] Dubrovskii V G, Sibirev N V, Cirlin G E, Bouravleuv A D, Samsonenko Yu B, Dheeraj D L, Zhou H L, Sartel C, Harmand J C, Patriarche G and Glas F 2009 Role of non-linear effects in nanowire growth and crystal phase Phys. Rev. B 80205305

[24] Harmand J C, Glas F and Patriarche G 2010 Growth kinetics of a single $\mathrm{InP}_{1-\mathrm{x}} \mathrm{As}_{\mathrm{x}}$ nanowire. Phys. Rev. B 81235436

[25] Glas F 2010 Vapor fluxes on the apical droplet during nanowire growth by molecular beam epitaxy Phys. Stat. Sol. B 247 254-258

[26] Glas F, Ramdani M R, Patriarche G and Harmand J C 2013 Predictive modeling of selfcatalyzed III-V nanowire growth Phys. Rev. B 88195304

[27] Dubrovskii V G 2016 Group V sensitive vapor-liquid-solid growth of Au-catalyzed and selfcatalyzed III-V nanowires. J. Cryst. Growth 440 62-68

[28] Hairer E, Norsett S P and Wanner G 1993 Solving ordinary differential equations II: stiff and differential-algebraic problems, 2nd ed. (Berlin Heidelberg: Springer-Verlag)

[29] Virtanen P, Gommers R, Oliphant T E, Haberland M, Reddy T, Cournapeau D, Burovksi E, Peterson P, Weckesser W, Bright J, van der Walt S J 2020 SciPy 1.0: fundamental algorithms for computing in Python Nat. Methods 17 261-272

[30] Storn R, Price K 1997 Differential evolution - a simple and efficient heuristic for global optimization over continuous spaces J. Glob. Optim. 11 341-359 
[31] Goktas N I, Sokolovskii A, Dubrovskii V G, LaPierre R R 2020 Formation mechanism of twinning superlattices in doped GaAs nanowires Nano Lett. 20 3344-3351 